\title{
Ultraviolet photoproduction of ISM dust
}

\section{Laboratory characterisation and astrophysical relevance}

\author{
E. Dartois ${ }^{1}$, G. M. Muñoz Caro ${ }^{1}$, D. Deboffle ${ }^{1}$, G. Montagnac ${ }^{2}$, and L. d'Hendecourt ${ }^{1}{ }^{1} \star$ \\ 1 Institut d'Astrophysique Spatiale, UMR - 8617, Université Paris-Sud, Bâtiment 121, 91405 Orsay Cedex, France \\ e-mail: emmanuel.dartois@ias.u-psud.fr \\ 2 Laboratoire des Sciences de la Terre, UMR 5570 CNRS, École Normale Supérieure de Lyon, 46 allée d'Italie, \\ 69364 Lyon Cedex 07, France
}

Received 30 September 2004 / Accepted 30 October 2004

\begin{abstract}
The production of a hydrogenated amorphous carbon polymer $(\mathrm{a}-\mathrm{C}: \mathrm{H})$ via the photolysis of a series of organic molecule precursors at low temperature is described. Such amorphous material is synthesised under interstellar conditions (10 K and Lyman- $\alpha$ photons) and represents the best candidate to explain the Diffuse Interstellar Medium absorption observed in our Galaxy and in other galaxies. We perform a series of laboratory analyses (Infrared spectroscopy, $\mu$ spectroscopy, Raman, Photoluminescence and UV-visible spectroscopy) which allow a full characterisation of such polymers. This allows us to assess the importance of the polymer and possible scenarios for its role in crucial aspects of the lifecycle of dust. Such material has implications for the carbon budget at galactic scales, hydrogen formation, extended red emission, as a PAH precursor, and in explaining the $2175 \AA$ A extinction bump.
\end{abstract}

Key words. ISM: dust, extinction - galaxies: ISM - ISM: lines and bands

\section{Introduction}

The composition of the diffuse interstellar medium dust observed in extinction is a subject of long-standing debate which has led to an impressive amount of astrophysical literature. The $3.4 \mu \mathrm{m}$ absorption feature observed against galactic center background sources has received particular attention since its discovery in the early eighties (Allen \& Wickramasinghe 1981). It soon appeared that this feature should be associated with methyl $\left(-\mathrm{CH}_{3}\right)$ and methylene $\left(-\mathrm{CH}_{2}-\right)$ group stretching mode absorptions in carbonaceous dust (Duley \& Williams 1983) and numerous experiments and observations were conducted to constrain its origin (e.g. Mennella et al. 2002; Pendleton \& Allamandola 2002; Chiar et al. 2002; Geballe et al. 1997; Tielens et al. 1996; Sandford et al. 1995; Pendleton et al. 1994; Sandford et al. 1991; Ehrenfreund et al. 1991; McFadzean et al. 1989; Butchart et al. 1986; Jones et al. 1983; Moore \& Donn 1982, and references in these articles). Extension of observations by space observatories (Infrared Space Observatory or Spitzer Space Telescope), with low to moderate spectral resolution, to the mid infrared spectra of lines of sight where the $3.4 \mu \mathrm{m}$ feature is observed, gave insight into additional dust solid state bands at 6.85 and $7.25 \mu \mathrm{m}$ (e.g. Chiar et al. 2000; Spoon et al. 2004), but also

* Based on Experiments performed at IAS-CNRS and observations from ESO-VLT and Spitzer Space Telescope. point to the absence of large quantities of oxygen in these dust features (Dartois et al. 2004a).

Recent observations shed light on the fact that Galactic diffuse interstellar dust features are common in external galaxies (Imanishi 2000; Spoon et al. 2002). They are ubiquitous galactic and extragalactic ISM features. This ISM dust is a large fraction (at least 5 to $30 \%$ of the carbon cosmic abundance, Duley et al. 1998; Sandford et al. 1991) of the matter that is important for the evolution of dust and ISM solid phase chemistry.

In this article we present an analysis of the ultraviolet production of an interstellar dust analog, which we call photoproduced a-C:H. This material is a parent of a plasma-produced analog, often called plasma a-C:H by physicists, but that astrophysicists call HAC (Hydrogenated Amorphous Carbon, e.g. Grishko \& Duley 2002; Furton et al. 1999; Scott et al. 1997; Duley 1994; Blanco et al. 1991; Jones et al. 1990). We will adopt the a-C:H nomenclature because, even though these materials are related, they do not refer to production methods with similar quantities of UV and ion irradiation.

\section{Experiments}

\section{Infrared}

The setup used to produce and analyse the samples in situ consists of a Bruker IFS66V infrared spectrometer coupled to a cryostat evacuated to pressure $<10^{-7}$ mbar. In this cryostat, at the end of a cold finger, the substrate is an infrared 
transmitting window, cooled to $10-77 \mathrm{~K}$ (using $\mathrm{LN}_{2}$ or $\mathrm{LHe}$ transfer). A-C:H production is performed by slow codeposition of an organic progenitor (in the gas phase at ambient temperature), through a deposition tube facing the substrate window, with simultaneous UV irradiation. The cooled infrared transparent substrate is made of KRS5, ZnSe, Ge or CsI, depending on the subsequent analysis to be performed. $\mathrm{ZnSe}$ was chosen as it is able to resist annealing temperatures of up to $\sim 700$ degrees Celsius under vacuum. CsI, KRS5 and Ge were selected for their increasing refractive index to obtain variations in the thin a-C:H film interference fringe amplitudes to estimate the optical constant of the material. The UV irradiation is performed with an Evenson cavity used to excite microwave discharges in molecular hydrogen with a $2450 \mathrm{MHz}$ microwave generator. The hydrogen plasma is interfaced to the cryostat via an $\mathrm{MgF}_{2}$ window, efficiently transmitting the produced UV photons up to $1200 \AA$. A typical flux of $5 \times 10^{14}$ photons s $\mathrm{s}^{-1} \mathrm{~cm}^{-2}$ impinginges on the film during deposition. Given that the DISM photon field is $\sim 8 \times 10^{7}$ photons $\mathrm{s}^{-1} \mathrm{~cm}^{-2}$ (Mathis et al. 1983), this corresponds to roughly two to three months of diffuse interstellar UV irradiation per second. We selected saturated (methane, ethane, hexane) and unsaturated (Trans-2-butene, 1,5-hexadiene) aliphatic species as molecular precursors, and xylene was used as representative of aromatic compounds (however possessing an aliphatic character due to the methyl groups). We studied the effect of including nitrogen in the aliphatic chain by using diethylamine and triethylamine precursors. The deposition/irradiation lasts about sixty hours to produce a film whose final thickness is about one to ten microns. Some of the films were prepared using a liquid helium transfer $(10 \mathrm{~K}$ for the precursor: methane, ethane, trans-2-butene), the others with liquid nitrogen ( $77 \mathrm{~K}$ for the precursor: hexane, 1,5-hexadiene, xylene - mixture of isomers -, triethylamine, diethylamine). The films are then annealed at room temperature to evaporate all the radicals and/or molecules trapped in the film structure and cooled again for infrared measurements with a resolution of $1 \mathrm{~cm}^{-1}$. Blank experiments were performed in which the experiment was carried without depositing any precursor, in order to ensure that no spurious contaminant was responsible for the production of the film. The main contamination in the film production arises from low amounts of residual water in the system, and in the spectra translates into the appearance of a small carbonyl contribution. Typical spectra of the intermediate temperature products were described in a short communication for the methane precursor case (Dartois et al. 2004b). The resulting film spectra are presented in Fig. 1 for pure $(\mathrm{a}-\mathrm{C}: \mathrm{H})$, and in Fig. 2 for the two (a-C:H:N), normalised to the optical depth of the $\mathrm{CH}$ stretching mode region.

After production, a series of other ex-situ analyses were performed on these films, described below.

\section{Infrared microspectroscopy}

FTIR microspectroscopy was performed with a Nicolet Magna-IR 560 ESP spectrometer coupled to a Nicolet Nicplan infrared microscope, located on the SU5 line at the Laboratoire

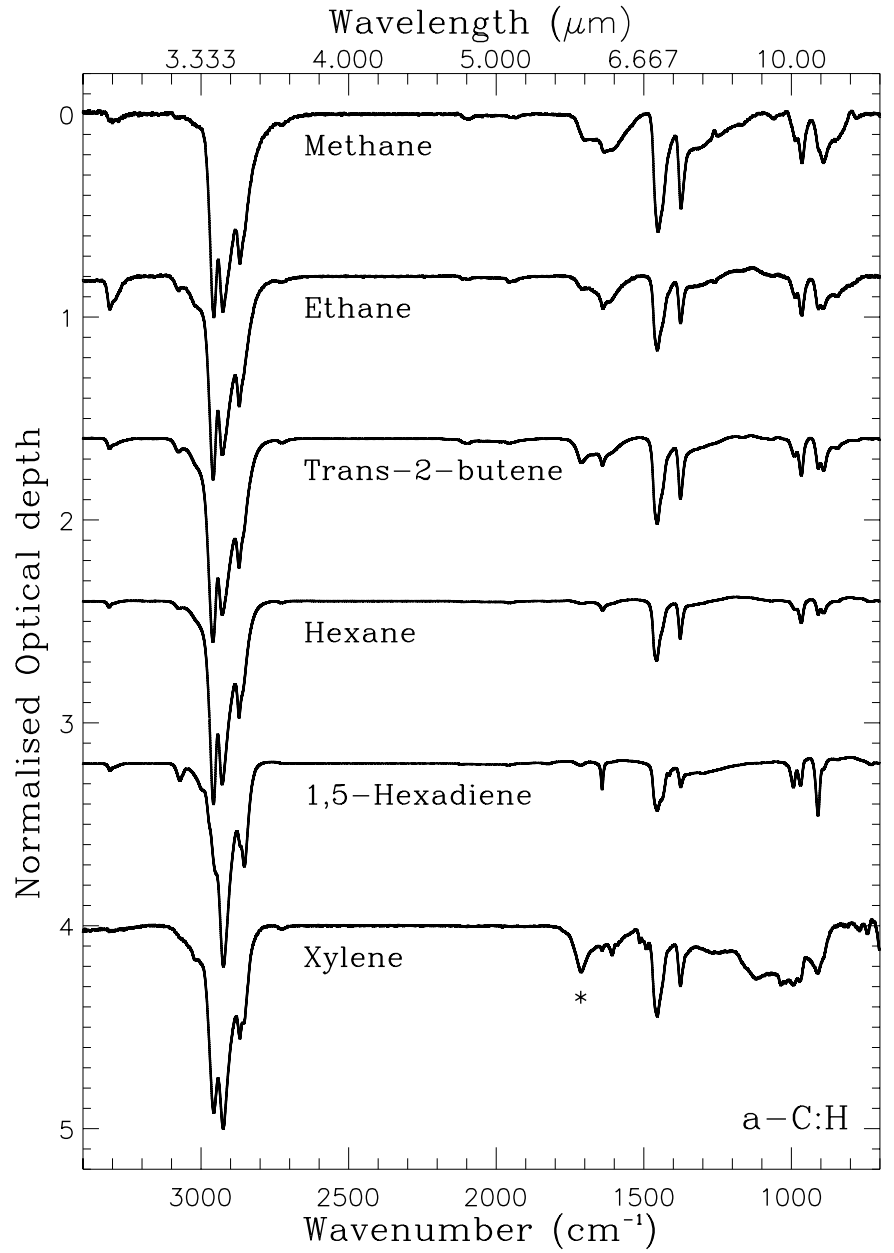

Fig. 1. Baseline corrected optical depth spectra of photoproduced a-C:H, normalised to the stretching mode absorption region for comparison. Each spectrum is labeled with the precursor from which the polymer originates. The vibrational assignment and discussion of the various features are detailed in the text.

pour l'Utilisation du Rayonnement Electromagnétique (LURE) at the University of Paris-Sud, Orsay, France. The spectrometer is equipped with a $\mathrm{KBr}$ beamsplitter and a nitrogen-cooled mercury-cadmium-tellure (MCT) detector working in the 4000 to $650 \mathrm{~cm}^{-1}$ (2.5 to $\left.15 \mu \mathrm{m}\right)$ spectral range. The microscope uses a Schwarzchild-Cassegrain objective $(32 \times)$ and a condenser ( $10 \times$ in the transmission mode). We used the internal standard light source of the instrument (Globar), which is stable and allows spectroscopic analysis of samples with sizes larger than $\sim 6 \mu \mathrm{m}$ (Raynal et al. 2000).

\section{Raman spectroscopy and associated fluorescence}

The residues were also analysed in Lyon with one of the Raman spectrometers of the Laboratoire de Sciences de la Terre (Labram HR800 vis Jobin Yvon, coupled to a Spectra Physics argon ion laser tuned for these experiments into the green $(514.5 \mathrm{~nm})$ or blue $(457.9 \mathrm{~nm})$ part of the visible spectrum). The laser beam is focused through microscope objectives $(\times 50)$ down to a $2 \mu \mathrm{m}$ spot on samples, and the backscattered light is collected through the same objective. For Raman 


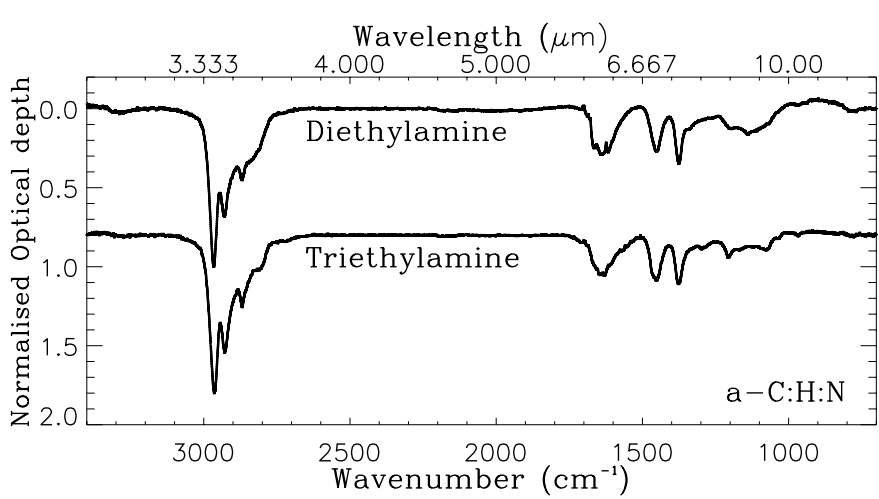

Fig. 2. Baseline-corrected optical depth spectra of photoproduced a-C:H:N, normalised to the stretching mode absorption region for comparison. Each spectrum is labeled with the precursor from which the polymer originates. The principal additional vibrations expected from the presence of the nitrogen are labeled and detailed in the text.

spectra, a 1800 grooves per millimeter grating was used in a wavelength range centered around the $\mathrm{D}$ and $\mathrm{G}$ carbonaceous lines (Raman shift $\sim 1600 \mathrm{~cm}^{-1}$ ). Wavelength calibration was checked against a silicon wafer sample, and agreed to within better than $1 \mathrm{~cm}^{-1}$ for all the experiments. To record the luminescence, we used a 600 groves per millimeter grating, and the grating position varied during exposure to the laser beam to cover a wide wavelength range. Individual subscans were in that case stitched together, applying a gain factor estimated in the overlapping region. In both Raman and fluorescence modes, care was taken to irradiate the sample with low power fluxes (typically $50 \mu \mathrm{W}$ ), so as not to modify its structure, by interposing neutral optical density filters (OD from 2 to 4 ), as it was noticed that without such densities, exposures to $\mathrm{mW}$ power made the film evolve to more aromatic spectra (graphitisation).

\section{UV-visible spectroscopy}

UV-visible spectra of a dedicated a-C:H very thin film ( $\sim 0.06 \mu$ m thick) deposited onto a $\mathrm{CaF}_{2}$ transmitting window were recorded in transmittance, using a CARY 5 spectrophotometer, from $190 \mathrm{~nm}$ to $1000 \mathrm{~nm}(\sim 1.23-6.7 \mathrm{eV})$, at a resolution of $1 \mathrm{~nm}$, with a beam on the sample of about $8 \mathrm{~mm}$ height and $5 \mathrm{~mm}$ wide.

\section{Infrared spectroscopy}

\section{1. a-C:H vibrational assignment}

The assignment of the prominent bands observed in the film spectra presented in Fig. 1 are given in Table 1 for the $\mathrm{a}-\mathrm{C}: \mathrm{H}$ and the additional vibrations arising from the presence of nitrogen in a-C:H:N in Table 2. These identifications are based on work done on plasma-produced a-C:H (e.g. Ristein et al. 1998, and references therein) and selected studies of polymers containing aliphatic/olefinic characters (e.g. Nava et al. 1996; Bourée et al. 1996). Films produced from methane, trans-2-butene, ethane and hexane display very similar spectra and therefore the same underlying structure. In plasma production, this is often refered to as the "lost memory
Table 1. a-C:H vibrational assignments (see text for details)

\begin{tabular}{|c|c|}
\hline Position $\left(\mathrm{cm}^{-1}\right)$ & Vibrational mode \\
\hline 3300 & alkyne CH str. \\
\hline 3078 & olefinic $\mathrm{CH}_{2}$ asym.str. \\
\hline 3050 & aromatic $\mathrm{CH}$ \\
\hline 3010 & olefinic $\mathrm{CH}$ \\
\hline 2985 & olefinic $\mathrm{CH}_{2}$ sym.str. \\
\hline 2960 & aliphatic $\mathrm{CH}_{3}$ asym.str. \\
\hline 2925 & aliphatic $\mathrm{CH}_{2}$ asym.str. \\
\hline 2900 & aliphatic $\mathrm{CH}_{2}$ Fermi resonance ${ }^{a}$ \\
\hline 2871 & aliphatic $\mathrm{CH}_{3}$ sym.str. \\
\hline 2850 & aliphatic $\mathrm{CH}_{2}$ sym.str. \\
\hline 2100 & alkyne $\mathrm{CH}$ bend. \\
\hline 1900 & $\mathrm{C}=\mathrm{C}=\mathrm{C}$ bend. \\
\hline$\sim 1700$ & carbonyl contamination \\
\hline 1640 & olefinic $\mathrm{C}=\mathrm{C}$ \\
\hline 1600 & aromatic $\mathrm{C} ;=\mathrm{C}$ \\
\hline 1470 & aliphatic $\mathrm{CH}_{3}$ asym.bend. \\
\hline 1450 & aliphatic $\mathrm{CH}_{2}$ bend. \\
\hline 1430 & aromatic $\mathrm{CH}$ bend. \\
\hline 1415 & olefinic $\mathrm{CH}_{2}$ bend. \\
\hline 1400 & olefinic $\left(\mathrm{CH}_{3}\right)_{3}$ sym.bend. \\
\hline 1370 & aliphatic $\mathrm{CH}_{3}$ sym.bend. \\
\hline $1100-1400$ & skeletal C, olefinic rockings \\
\hline 990 & $-\mathrm{CH}=\mathrm{CH}_{2}$ \\
\hline 970 & $-\mathrm{CH}=\mathrm{CH}-$ \\
\hline 910 & $-\mathrm{CH}=\mathrm{CH}_{2}$ \\
\hline 890 & $>\mathrm{CH}=\mathrm{CH}_{2}$ \\
\hline 845 & $>\mathrm{CH}=\mathrm{CH}-$ \\
\hline
\end{tabular}

${ }^{a}$ Discussed in a later section.

Table 2. Some expected additional vibrations in a-C:H:N.

\begin{tabular}{ccc}
\hline \hline Position $\left(\mathrm{cm}^{-1}\right)$ & Vibrational mode & Presence \\
\hline $3300-3500$ & $\mathrm{NH}$ stretching & weak \\
$2150-2200$ & $-\mathrm{C} \equiv \mathrm{N} / \mathrm{N} \equiv \mathrm{C}$ stretching & absent \\
$1610-1660$ & Imine $(>\mathrm{C}=\mathrm{N}-)$ stretching & present \\
$1020-1280$ & $\mathrm{C}-\mathrm{N}$ stretching & present \\
\hline
\end{tabular}

effect", because the precursor used to produce the film cannot be determined from the resulting spectrum. However, this effect is less pronounced for the photoproduced films presented here than for the radio frequency discharge produced ones. For example, the polymer based on hexadiene displays a spectrum similar to the other aliphatic precursors, but it is evident from the higher absorbance ratio of $-\mathrm{CH}_{2}$ - to $-\mathrm{CH}_{3}$ related vibrations that it has conserved a more chain-like character (Fig. 1). The film produced using a mixture of xylene isomers possesses a 
stronger 900 to $1500 \mathrm{~cm}^{-1}$ absorption which indicates a more complex carbon skeleton structure, containing a more aromatic network. The efficiency of formation of this polymer was lower than the others by about an order of magnitude, revealing also that the important basic ingredients for the propagation of a network are related to the radicals produced from methyl and methylene groups as well as the terminations opened when an $\mathrm{H}$ atom is ejected.

\section{2. a-C:H:N, effect of nitrogen addition}

Given that nitrogen is about one third to one fourth of the interstellar atomic carbon abundance (e.g. Snow \& Witt 1996), the influence on the spectrum of the presence of nitrogen atoms in the structure was evaluated. For this we performed experiments irradiating diethylamine and triethylamine precursors, displayed in Fig. 2.

There are various ways to include nitrogen in the amorphous carbon polymer network. $\mathrm{C}-\mathrm{N}$ gives rise to a certain level of underlying absorption in the $1280-1020 \mathrm{~cm}^{-1}$ region. However, the most prominent band appears in the $1610-1660 \mathrm{~cm}^{-1}$ region in both spectra, which corresponds to the $\mathrm{C}=\mathrm{N}$ imine group (Yoon et al. 1999). The low $\mathrm{NH}$-stretching vibrations contribution in the $3300-3500 \mathrm{~cm}^{-1}$ range probably reflects the ease of photochemical breaking of the $\mathrm{NH}$ bond. No strong nitrile $\mathrm{C} \equiv \mathrm{N}$ or isonitrile $\mathrm{N} \equiv \mathrm{C}$ absorptions appear in the $2150-2200 \mathrm{~cm}^{-1}$ window, even if it is present at a low level in the photolysed diethylamine spectrum. This suggests that the route of formation is not energetic enough to overcome the formation barrier for these triple bonds, unlike the a-C:H:N produced with high energy ions in some plasmas (Motta \& Peyeyra 2004; Lazar \& Lazar 2003; Vyskocil et al. 1996).

The inclusion of nitrogen in the polymeric network of a-C:H:N produced from photolysis of a precursor seems to favour an non-nitrile phase inclusion. More specifically, the high level of imine contribution shows that nitrogen acts as a bridge in the network between the aromatic clusters, but does not seem to be included as a heteroatom in the aromatic part of the network.

\subsection{Methylene Fermi resonance}

The decomposition of the a-C:H stretching mode absorption profile into individual characteristic group vibrations gives insight into the relative proportions of $\mathrm{sp}^{3}, \mathrm{sp}^{2}$ and $\mathrm{sp}^{1}$ hybridized carbons, provided that these carbon atoms are linked to at least one hydrogen atom (i.e. dominated neither by diamond nor graphene phases). The asymmetric $\mathrm{CH}_{3}$ stretching mode around $2960 \mathrm{~cm}^{-1}$ can be reasonably fitted by a Gaussian profile (Dartois et al. 2004b). However, the asymmetric $\mathrm{CH}_{2}$ mode $\left(\sim 2920 \mathrm{~cm}^{-1}\right)$ presents an asymmetry that requires an additional red wing contribution around $2900 \mathrm{~cm}^{-1}$. This mode is often erroneously attributed to an $\mathrm{sp} 3$ diamond-like $\mathrm{CH}$ stretching mode, whereas its contribution should be low for highly hydrogenated polymeric material (Ristein et al. 1998).

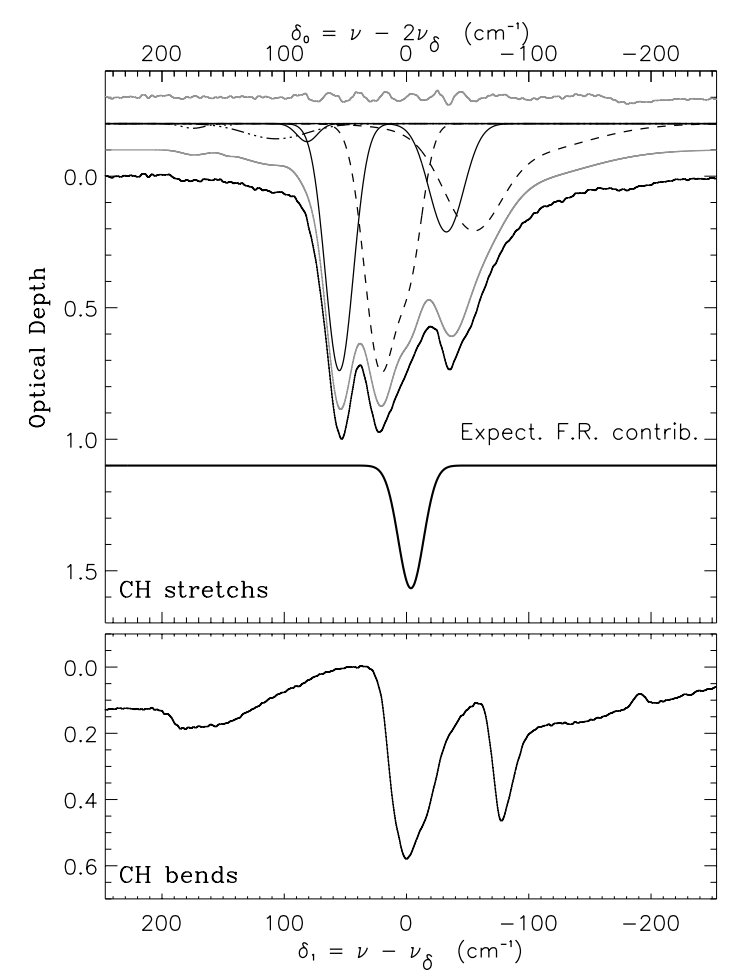

Fig. 3. Upper panel: stretching mode region of the a-C:H produced with methane as a precursor, together with the typical fit decomposition with Gaussians discussed in Dartois et al. (2004b). This frequency window is shown against the frequency difference $\left(\delta_{0}=v-2 v_{\delta}\right)$ with respect to the second harmonic of the $\mathrm{CH}_{2}$ bending mode $2 v_{\delta}$, which lies around $2 \times 1452 \mathrm{~cm}^{-1}$. The additional line at $\sim 2900 \mathrm{~cm}^{-1}$, required in the profile decomposition and corresponding to the Fermi resonance, is presented just below. In the lower panel, the $\mathrm{CH}_{2}$ bending mode region is displayed against the frequency difference $\left(\delta_{1}=\right.$ $v-v_{\delta}$ ) with respect to the fundamental $\mathrm{CH}_{2}$ bending mode $v_{\delta}$, for comparison.

We propose that the peculiar profile of the stretching mode region of amorphous- $\mathrm{C}: \mathrm{H}$ polymers is the consequence of a Fermi resonance between the $\mathrm{CH}_{2}$ symmetric stretching mode of methylene groups and the bending scissor vibration $\left(v_{\delta}\right)$, with, as a consequence, borrowing of intensities between these modes.

The Fermi resonance arises when the overtone of a vibrational mode of a molecule falls near the frequency of another fundamental mode, giving rise to a perturbation splitting. In Fig. 3, upper panel, we show the stretching mode region of the a-C:H produced with methane as a precursor, together with the typical fit decomposition with Gaussians already discussed in Dartois et al. (2004b). This region is shown against the frequency difference with respect to the second harmonic of the $\mathrm{CH}_{2}$ bending mode $2 v_{\delta}$, which lies around $2 \times 1452 \mathrm{~cm}^{-1}$, to better appreciate the overlap in frequency with the bending mode overtone. The additional line at $\sim 2900 \mathrm{~cm}^{-1}$ that was required in the profile decomposition is presented just below. In the lower panel, the $\mathrm{CH}_{2}$ bending mode region is displayed against the frequency difference with respect to the fundamental $\mathrm{CH}_{2}$ bending mode $v_{\delta}$, for comparison. 


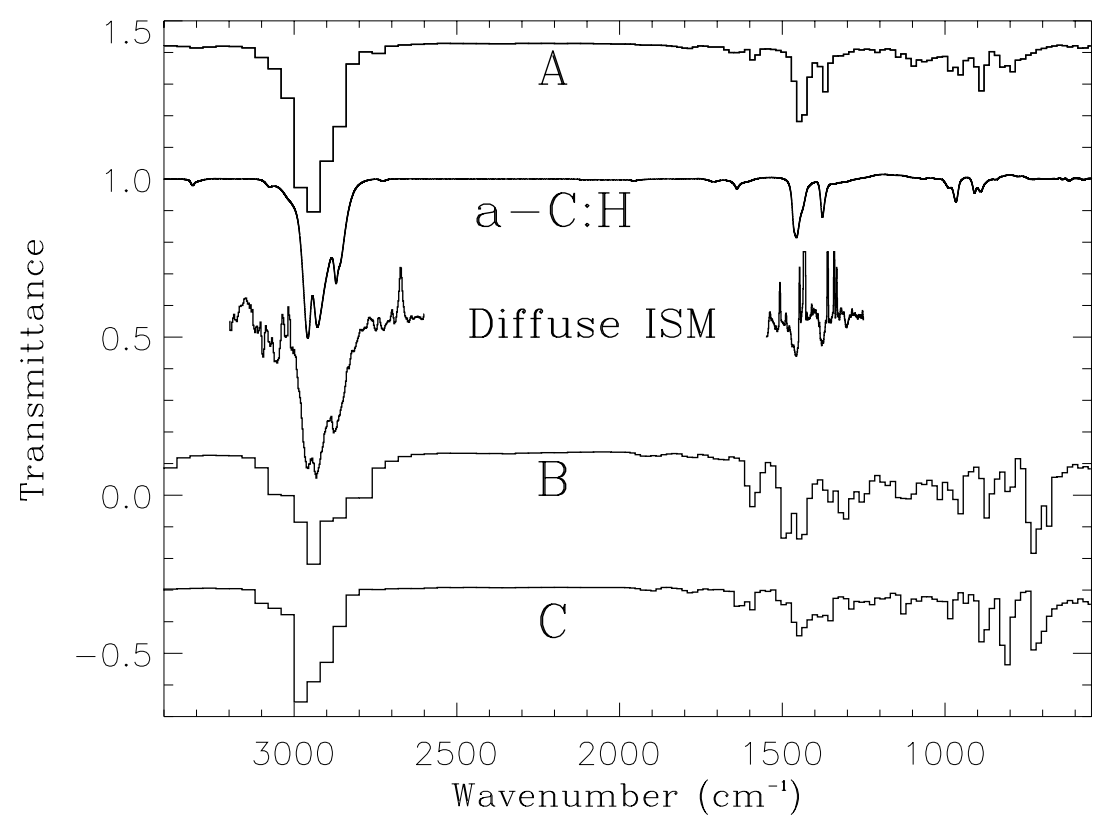

Fig. 4. Simulations based on neuronal networks (Selzer et al. 2000) of the infrared spectra of large polymer-like structures: A) expected photoproduced a-C:H unit substructure, compared to the laboratory spectra of a-C:H made from hexane. The diffuse ISM observed spectrum is also presented for comparison (Dartois et al. 2004b). In this spectrum, the high frequency narrow peaks are attributed to emission lines arising near the Galactic center; B) and C) substructures presented in Pendleton \& Allamandola (2002). All transmittances are in arbitrary units to allow

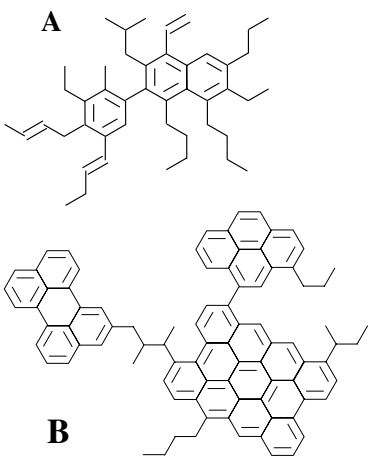
spectral comparison.

The Fermi resonance is what we suggest to explain the red wing of the $\mathrm{CH}_{2}$ asymmetric vibration. Such a resonance has been discussed in the litterature, both theoretically and experimentally (e.g. Snyder \& Scherer 1979; Manner et al. 1998; Ratajczak \& Yaremko 1999; Jordanov et al. 2003) in other cases, and is expected to contribute significantly for alkyl chains around $\sim 2900 \mathrm{~cm}^{-1}$. This Fermi resonance indicates the difficulty in analysing the spectral profile and band intensities of complex organic materials using the simple view of independent vibrations.

\subsection{Structure estimate of a-C:H}

In order to estimate the olefinic to aliphatic ratio in the structure produced, we used features that are sufficiently separated in frequency to be isolated by a fitting procedure. Good candidates are the asymmetric stretching mode of the olefinic $\mathrm{CH}_{2}\left(\sim 3078 \mathrm{~cm}^{-1}\right)$ and the asymmetric stretching mode of aliphatic $\mathrm{CH}_{3}\left(\sim 2960 \mathrm{~cm}^{-1}\right)$. Their cross sections have been evaluated and given in e.g. Ristein et al. (1998). Their ratio, $\sigma$ (olef.asym. $\left.\mathrm{CH}_{2}\right) / \sigma\left(\right.$ aliph.asym. $\left.\mathrm{CH}_{3}\right)$ is about 0.55 . We therefore deduce that our films contain 5 to $10 \%$ olefinic $\mathrm{CH}_{2}$ bonding with respect to the aliphatic $\mathrm{CH}_{3}$. The same calculation of the aliphatic $\mathrm{CH}_{2} / \mathrm{CH}_{3}$ asymetric modes gives a ratio of about two, although the presence of a Fermi resonance discussed in the previous section does not allow a better ratio estimate as it will somewhat influence the band integrated absorbances. The aromatic contribution is the most difficult to assess as the only suitable band is the $\mathrm{C}=\mathrm{C}$ stretch overlapped by the olefinic $\mathrm{C}=\mathrm{C}$. Given that the intensities of olefinic and aromatic $\mathrm{C}=\mathrm{C}$ transitions should be within a factor of two (Wexler 1967), we expect from 5\% to at most $20 \%$ aromatic $\mathrm{C}=\mathrm{C}$ in the a-C:H network.

\subsection{Neuronal network simulations}

An interesting recent approach to predict infrared spectra was developed using neuronal networks (Selzer et al. 2000). Such a method allows rapid access to infrared reference spectra of arbitrary organic compounds. The neural networks learn from experimental data about the correlation of structure and spectra by analyzing a set of examples. By presenting a new structure to a trained neural network, the learned correlation can be used to predict an infrared spectrum for an unknown compound.

We made use of these infrared simulations ${ }^{1}$ to calculate the spectra of the large molecular structures presented in Fig. 4 and compare them to our produced polymer spectra. We especially focused our attention on comparing the molecular structure we believe to be relevant to interpret the aspects of photoproduced $\mathrm{a}-\mathrm{C}: \mathrm{H}$ to molecular structures encountered in the astrophysical literature. The structure $\mathbf{A}$ was designed on the basis of what we deduced from the previous analyses and, except for the small excess around $800 \mathrm{~cm}^{-1}$, the spectra are equivalent. The two other structures ( $\mathbf{B}$ and $\mathbf{C}$ ) were chosen based on the generally accepted structure of carbonaceous interstellar dust (e.g. Pendleton \& Allamandola 2002). Such networks do not allow

\footnotetext{
1 http://www2 .chemie.uni-erlangen.de/services/ telespec/
} 


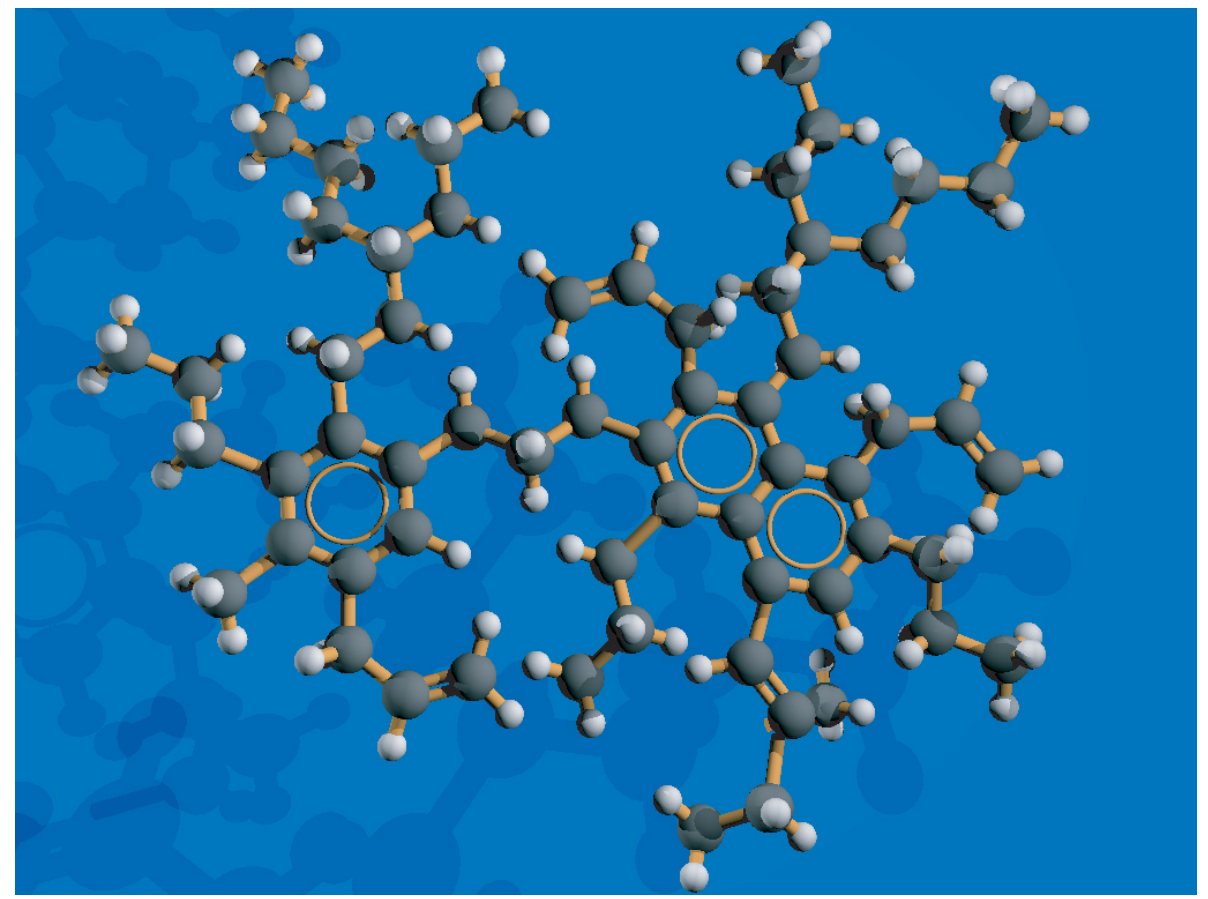

Fig. 5. A typical expected substructure unit for the hydrogen-rich photoproduced a-C:H. See also Fig. 1 of Jones et al. (1990).

a fit to the observed ISM spectra, as they display too many unobserved absorptions in the mid-infrared range.

Thus, we propose a substructure unit for the photoproduced a-C:H presented in Fig. 5. This is in perfect agreement with the many experimental and theoretical works on plasma-produced a-C:H (e.g. Robertson 1995; Jacob \& Moller 1993), but with a much higher hydrogen to carbon ratio.

\subsection{Optical constants of a-C:H}

The a-C:H film produced by the photochemistry of hexane was analysed by infrared microspectroscopy. The injection of the hexane precursor was made onto a $\mathrm{ZnSe}$ window making an angle with the deposition line. As a result, the photochemically produced film presents variations of thickness depending on its distance to this line. We used the resulting thickness gradient to select three clean regions with local high quality planeparallel film properties of $\sim 100 \mu \mathrm{m} \times 100 \mu \mathrm{m}$, and different thicknesses, to record their spectra. These spectra, presented in Fig. 6, display the characteristic polymer absorption features superimposed on the interference fringe patterns resulting from the intrinsic local film thickness. The equations defining the transmission of an absorbing film on a thick finite transparent substrate, in the absorption-free region of the spectra, allow us to deduce the film refractive index $n_{\mathrm{F}}$ (Swanepoel 1983) using the equation:

$$
\begin{aligned}
\Delta I & =\left(1 / T_{\mathrm{M}}-1 / T_{\mathrm{m}}\right)=\frac{\left(n_{\mathrm{F}}^{2}-1\right)\left(n_{\mathrm{F}}^{2}-n_{\mathrm{S}}^{2}\right)}{4 n_{\mathrm{F}}^{2} n_{\mathrm{S}}} \\
& \Rightarrow n_{\mathrm{F}}=\sqrt{\frac{\gamma \pm \sqrt{\gamma^{2}-4 n_{\mathrm{S}}^{2}}}{2}} \text { with } \gamma=\left(1+n_{\mathrm{S}}^{2}+4 n_{\mathrm{S}} \Delta I\right)
\end{aligned}
$$

where $n_{\mathrm{S}}$ is the ZnSe substrate refractive index, adopted from Hawkins 1998 and $T_{\mathrm{M}}$ and $T_{\mathrm{m}}$ are two adjacent maximum and minimum in the transmittance, respectively. The two roots of this equation at $\sim 2300 \mathrm{~cm}^{-1}\left(n_{\mathrm{F}} \approx 1.35\right.$ or 1.8$)$ are both valid without additional information.

The Kramers-Kronig relations between $n_{\mathrm{F}}$ and $k_{\mathrm{F}}$ imply that in the absence of nearby strong absorption, the refractive index of the film well above the $\mathrm{CH}$ stretching mode region $\left(>3200 \mathrm{~cm}^{-1}\right)$ should be lower than the one after it (around $\left.2300 \mathrm{~cm}^{-1}\right)$. In the spectra, we observe that $\Delta I\left(\sim 2300 \mathrm{~cm}^{-1}\right)>$ $\Delta I\left(\sim 3200 \mathrm{~cm}^{-1}\right)$, which, combined with $n_{\mathrm{F}}\left(\sim 2300 \mathrm{~cm}^{-1}\right)>$ $n_{\mathrm{F}}\left(\sim 3200 \mathrm{~cm}^{-1}\right)$ implies, given the dependence of $\Delta \mathrm{I}$ on $n_{\mathrm{F}}$ with the optical constants of $\mathrm{ZnSe}$, that the solution with $n_{\mathrm{F}}\left(\sim 2300 \mathrm{~cm}^{-1}\right) \approx 1.35$ is favoured.

When an absorption line is intrinsically of weak or of medium intensity, the refractive index of the film remains almost constant across this absorption band, meaning that no significant apparent absorption arises from reflections at the film interfaces in the band. One can then extract the "first order" absorbance spectrum by interpolating the absorption-free continuum from both sides of the absorption feature. By performing this analysis on the bending modes of each spectrum (see left panel of Fig. 6), we can establish and constrain the thickness ratios of the measured films. The modeled continua, expected without any absorption contribution, for the three thicknesses of polymer deposited on the $\mathrm{ZnSe}$ window, using the adopted refractive index, are displayed in Fig. 6 with dashed lines.

To validate the deduced refractive index, we perform another experiment in which we use a different substrate on which we produced the a-C:H film. We used a germanium substrate in order to drastically increase the refractive index of the substrate $\left(n_{\mathrm{S}} \sim 4\right)$ and therefore change the resulting fringe amplitudes. We analysed the film with the same microspectroscopic 

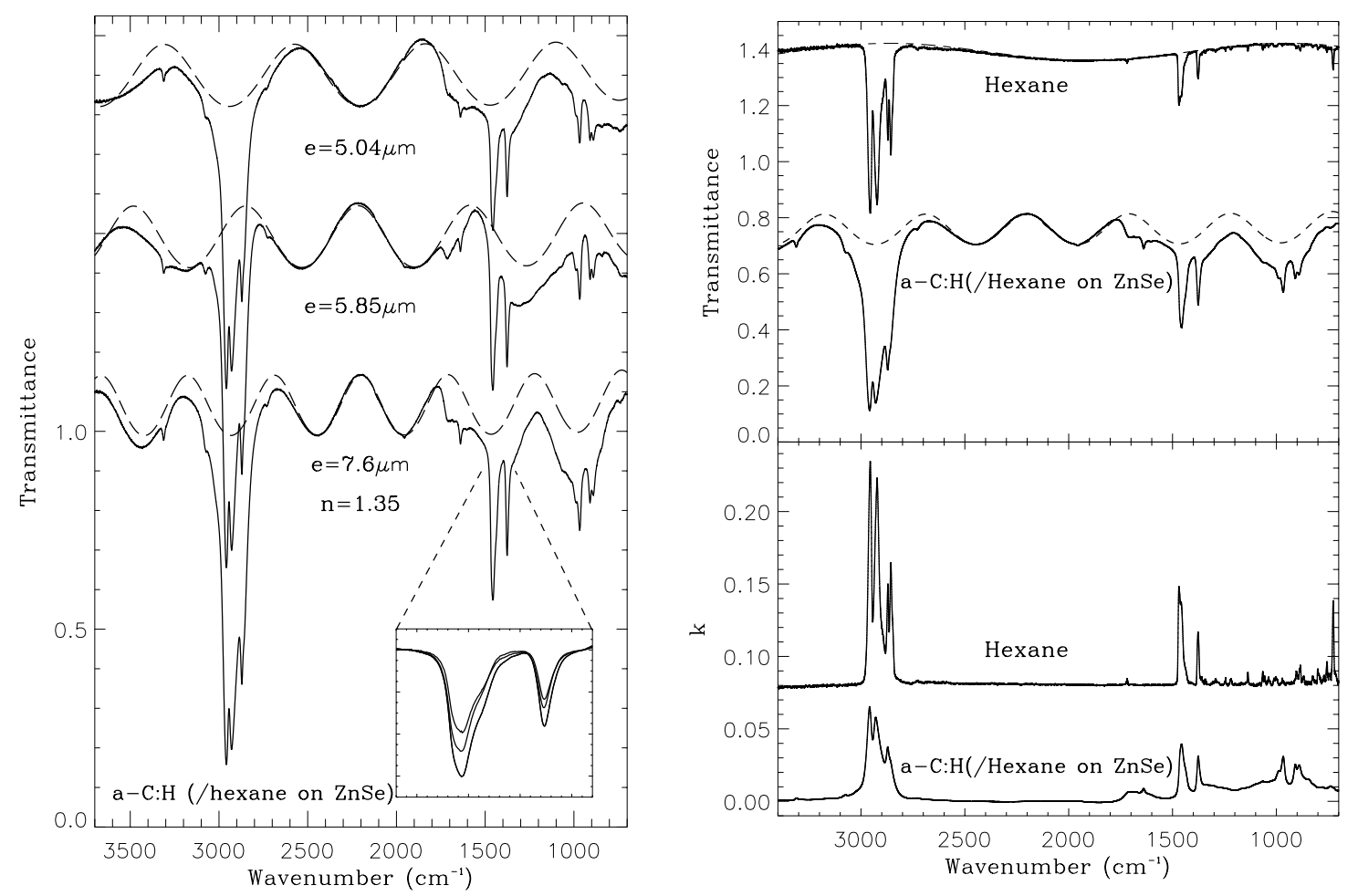

Fig. 6. Left panel: infrared microspectroscopy measurements of the transmittance of a-C:H produced from hexane on a $\mathrm{ZnSe}$ substrate. Three areas of $100 \times 100 \mu \mathrm{m}$, with different thicknesses, were measured to decouple interference patterns from pure absorptions of the polymer. The insert compares the different baseline-corrected transmissions of the three zones in the $\mathrm{CH}$ bending mode spectral region. This is used to estimate the true thickness ratios of the zones. Right panel: (upper) comparison between the transmittance of a pure ice hexane film deposited on CsI (Dartois et al. 2004a) and a-C:H (from hexane on ZnSe); (lower) comparison between the deduced imaginary index. See text for more details.

methods. Adopting the temperature-dependent refractive index of germanium measured by Hawkins 1998 we deduce a refractive index of $n_{\mathrm{F}}\left(\sim 2300 \mathrm{~cm}^{-1}\right) \approx 1.37$, in good agreement with the one obtained on the Zinc Selenide substrate. The modeled continua, expected without the absorption contribution, for the two different thicknesses of polymer deposited on the Ge window are displayed in Fig. 7 with dashed lines.

Other arguments are in favour of the attribution of the lower index. It has been shown for plasma-produced a-C:H that the refractive index decreases when the energy of the ions impinging on the film during its formation is lowered (energy proportional to an applied electrode bias voltage $V_{\mathrm{B}}$ ). Below $V_{\mathrm{B}} \approx$ $100 \mathrm{~V}$, this index is strictly lower than 1.8 (Koidl et al. 1990; Ristein et al. 1998). Based on the two independent measurements, we therefore deduce that the refractive index of this material is about $1.36 \pm 0.06$.

With the refractive index and thicknesses known, we can, to first order, use the baseline-corrected transmittance spectra to evaluate the imaginary optical constant $k$ of the material, using:

$k=-\frac{1}{4 \pi e \sigma} \operatorname{Ln}\left(\frac{\text { Tr measured }}{\text { Theoretical baseline }}\right)$

where $\sigma$ is the wavenumber of the line and $e$ the thickness of the film. The transmittances, modeled baselines and corresponding $k$ optical constants are given in Figs. 6 and 7 for both hexane ice deposited on a CsI window (Dartois et al. 2004a) and a-C:H produced from the photolysis of hexane ice onto a $\mathrm{ZnSe}$ and Ge window, for comparison.

The imaginary optical constant is lower than hexane for the a-C:H, especially in the $\mathrm{CH}$ stretching mode region.

This does not mean that the $\mathrm{CH}$ vibration constants are drastically affected, but rather that the material is porous (the low refractive index supports this view), and that other chemical groups participate in the film structure, as betrayed by the other $\mathrm{CH}$ infrared active modes and Raman spectra presented in a later section (a-C:H contains aromatic-like structures).

\section{Thermal annealing}

A series of annealing experiments was performed under high vacuum to evaluate the evolution of a-C:H under harsh conditions. The annealing proceeded in the following way: the $\mathrm{ZnSe}$ substrate on which the a-C:H film was previously deposited was placed in a glass tube and evacuated to a pressure below $10^{-6}$ mbar with a secondary pump. The temperature was monitored by a thermocouple. When the low pressure was reached, the tube was inserted into an oven at a fixed temperature for $30 \mathrm{~min}$ to allow thermalisation and then removed. Once the tube reached room temperature, the pumping was stopped and the sample analysed ex situ using microspectroscopy with an infrared beam of $\sim 100 \mu \mathrm{m} \times 100 \mu \mathrm{m}$. Care is taken to 


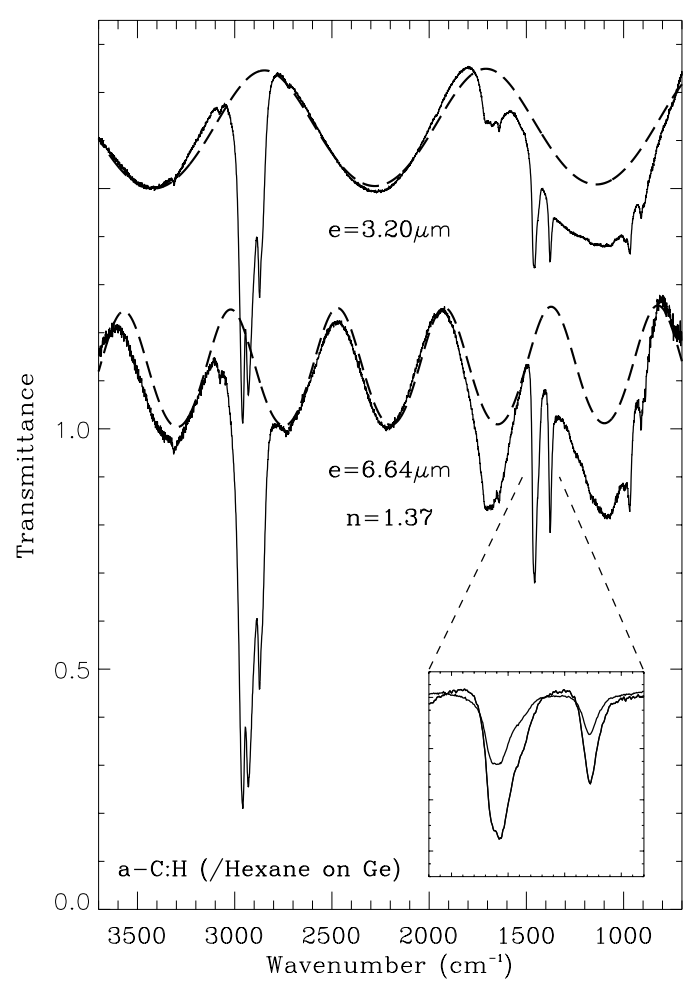

Fig. 7. Same as Fig. 6 for an a-C:H deposited on a germanium substrate.

analyse the same region of the substrate between two annealing steps using a CCD camera looking at the focal plane of the microscope. The results are then quantitative up to the level of uncertainty of the realignment of the infrared microscope and adopted aperture, i.e. to a few tens of percent. The annealing process is repeated in steps of $100 \mathrm{~K}$, starting at $700 \mathrm{~K}$, the temperature at which the main changes on the spectra are observed.

The results of the annealing are presented in Fig. 8 for the a-C:H(methane) and a-C:H(trans-2-butene). We describe the structure modification induced during annealing.

- $300 \mathrm{~K}$ to $700 \mathrm{~K}$ : the first sign of evolution is the loss of the olefinic character of the amorphous carbon network and the simultaneous appearance of aromatic-like structures. This behaviour is revealed by the disapperance of the olefinic $800-1000 \mathrm{~cm}^{-1}$ infrared absorptions and progressive conversion of the olefinic $\mathrm{C}=\mathrm{C} \sim 1640 \mathrm{~cm}^{-1}$ to aromatic $\mathrm{C}=\mathrm{C}$ $\sim 1600 \mathrm{~cm}^{-1}$. These changes operate principally in the heart of the network, as the aromatic $\mathrm{CH}$ stretching modes are not dominant. The reorganisation of the matrix gives rise also to a sharper carbonyl contamination peak, which is now distributed over more specific bonding sites. The network, however, still preserves its aliphatic character as the $\mathrm{CH}$ aliphatic stretching modes do not change drastically.

- $700 \mathrm{~K}$ to $800 \mathrm{~K}$ : here we see the real loss of the aliphatic character of the network, together with the carbonyl contaminant, which can therefore be assigned to aliphatic sites. This is an intermediate step between an aliphatic and aromatic character.

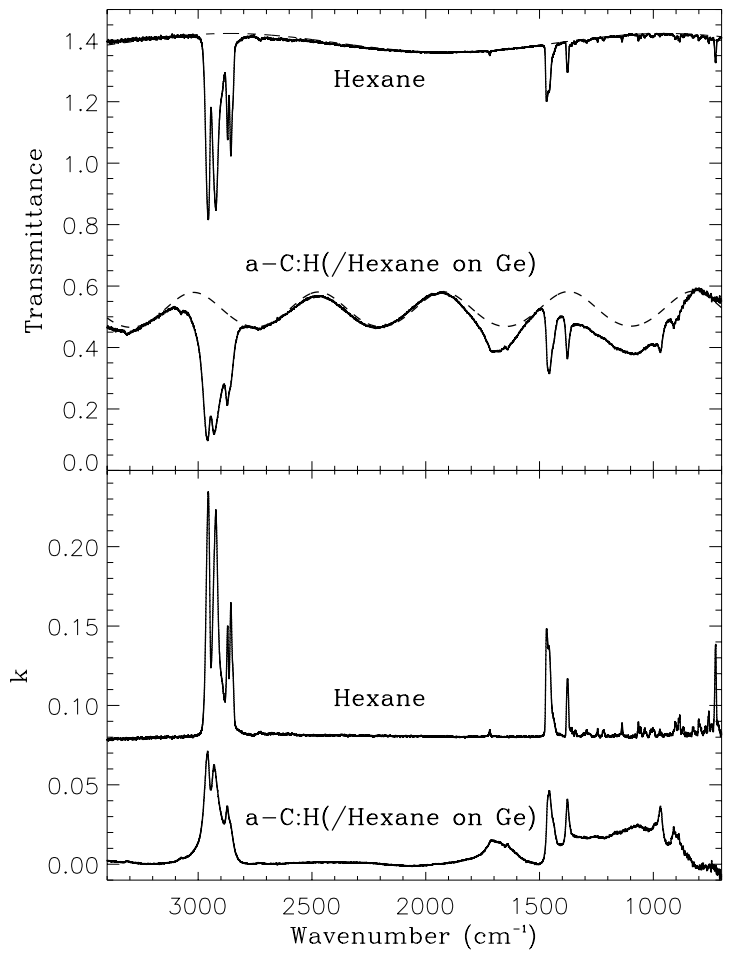

- $800 \mathrm{~K}$ to $900 \mathrm{~K}$ : most of the aliphatics have sublimated, and the isolated aliphatic chains have been replaced by hydrogen terminations, as the aromatic $\mathrm{CH}$ stretch reaches its maximum of the annealing steps. The same behaviour is observed for the $\mathrm{CH}$ out-of-plane vibrations, dominated by the solo stretch.

- $900 \mathrm{~K}$ to $1000 \mathrm{~K}$ : the evolution continues, progressively eliminating the $\mathrm{CH}$ bonds, and the material looks like graphene.

Thermal annealing favors the more stable configurations after a progressive elimination of the short chains in a-C:H. The consequent hydrogen abstraction leads to the formation of a polycyclic aromatic structure, ending with a graphite character when no hydrogen supply is added. In space, the evolution would be accompanied by rehydrogenation and the final step should be less graphite and more polyaromatic-like.

\section{Raman spectroscopy}

Raman spectroscopy of a-C:H is a way to probe the structure of the a-C:H carbonaceous backbone, and is complementary to the classical infrared method. This is a non-destructive technique, as long as the photon flow is sufficiently low. It is very important to control this effect when measuring Raman spectra of amorphous thin films, especially highly hydrogenated ones, as some published Raman plasma-produced a-C:H are in fact spectra of annealed film upon the application of the Raman laser (Lamberton et al. 1998).

Raman spectra of photoproduced a-C:H from hexane and trans-2-butene are displayed in Fig. 9 (lower spectra), from 

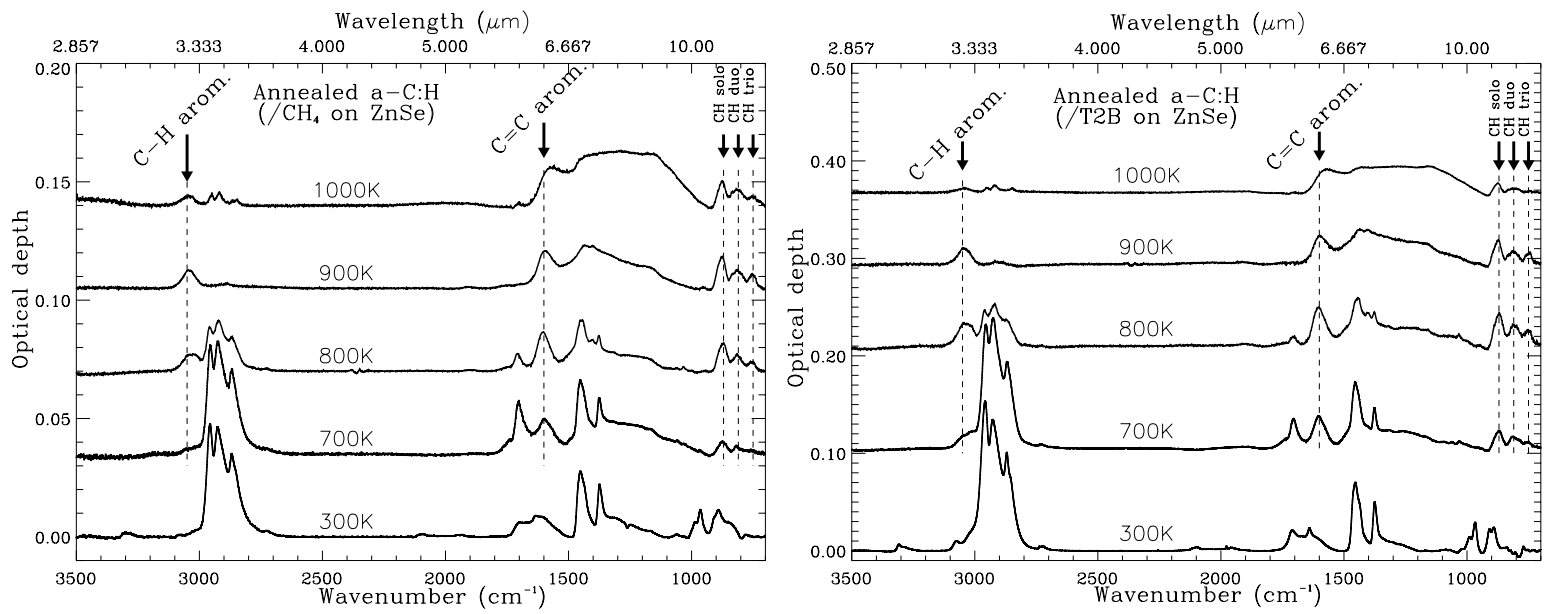

Fig. 8. Annealing experiments on a-C:H photoproduced with methane and trans-2-butene precursors, deposited on a ZnSe substrate, and evolution of their infrared spectra. Each curve is labeled with its corresponding annealing temperature, maintained during thirty minutes in the oven under vaccum $\left(<10^{-6} \mathrm{mbar}\right)$. The same $100 \times 100 \mu \mathrm{m}$ region of the film was each time recorded with an infrared $\mu$ scope after return to room temperature. The material progressively evolves from an aliphatic character to an aromatic one, as underlined by the onset of characteristic $\mathrm{C}=\mathrm{C}$ or $\mathrm{C}-\mathrm{H}$ absorptions features.

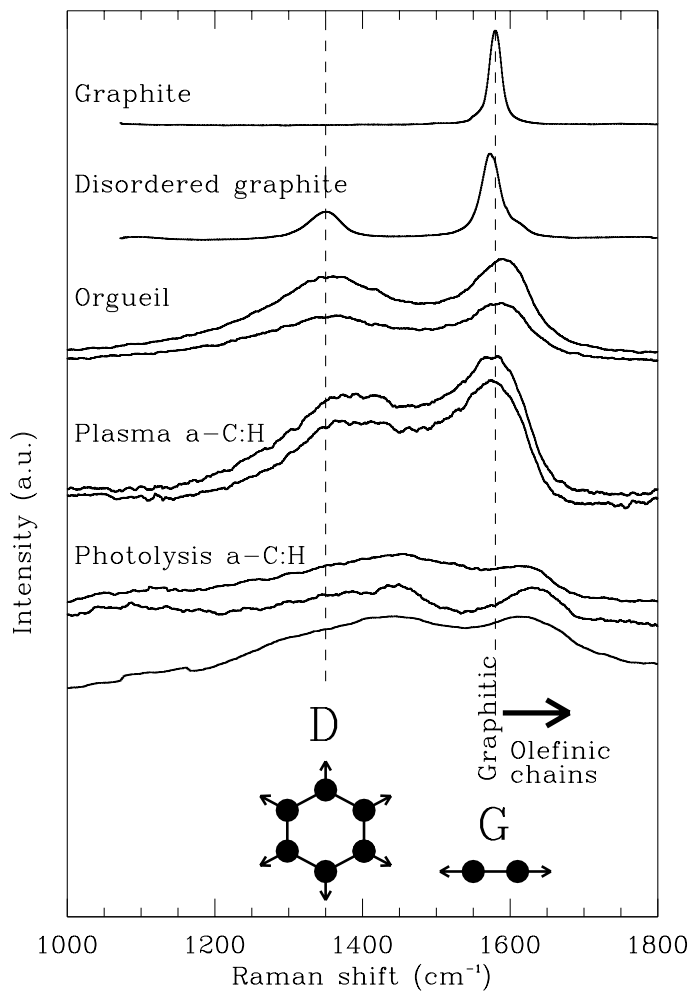

Fig. 9. Raman spectra of, from top to bottom: (i) graphite; (ii) disordered graphite (G. Montagnac, http: //www . ens-lyon. fr/ LST/Raman); (iii) Orgueil meteorite carbonaceous fraction; (iv) a$\mathrm{C}: \mathrm{H}$ produced in a hydrogen dominated RF plasma; (v) UV photoproduced a-C:H from hexane (two traces corresponding to two different spots on the same sample) and trans-2-butene precursors. The extraction of the last Raman spectra was very difficult due to the high photoluminescence background. The position of the $\mathrm{G}$ (badly labeled "graphitic" mode as it occurs in any $\mathrm{sp}^{2}$ paired carbon atoms) and D (breathing mode) in (disordered) graphite are given by vertical dashed lines. The arrow indicates the Raman G mode displacement in olefinic material. See text for further details. which we subtracted a second order baseline to remove the dominating luminescence spectrum of these polymers. They also have been smoothed to reduce the noise level. For comparison, a plasma a-C:H produced in our $\mathrm{H}_{2}$ discharge lamp and a chunk of the Orgueil meteorite organics are also presented, all measured on the same Raman spectrometer under the same conditions. We also added the graphite and disordered graphite measurements performed on the same instrument and extracted from the ENS laboratory database maintained by G. Montagnac ${ }^{2}$.

The materials presented in Fig. 9 share various degrees of aromatic ring components, organized differently. Two dominant bands are clearly observed. A so-called $G$ mode is seen around $1600 \mathrm{~cm}^{-1}$, badly labelled "Graphite" mode, which in fact is a $\mathrm{C}=\mathrm{C}$ stretching mode arising both in aromatic and olefinic organics. The second and large band in the $1300-1500 \mathrm{~cm}^{-1}$ range is more specific and corresponds to a breathing mode of aromatic rings. This mode, called D mode, is absent from pure graphite and related to the "Disorder" of the rings in the material. The $\mathrm{G}$ mode in photoproduced a-C:H lies at Raman shifts above $1600 \mathrm{~cm}^{-1}$, implying a non negligible contribution from olefinic $\mathrm{C}=\mathrm{C}$ bonds, whereas the other materials are dominated by aromatic carbon. The intensity ratio of the $\mathrm{D} / \mathrm{G}$ modes also gives insight into the structure. Based on previous studies (e.g. Ferrari 2002) and on the infrared information that tells us that the material also contains substantial olefinic bonds, a ratio of about 0.5 to 1 , as the one encountered in the measurements, implies few carbon ring structures of one to four rings, linked together. The similar intensity ratio for Orgueil does not have the same meaning for the underlying structure. Indeed, the function that relates $\mathrm{D} / \mathrm{G}$ intensity ratio and structure is not bijective. It is the position of the $\mathrm{G}$ peak, almost at the graphite location, that indicates larger polyaromatic structures in this case. The width of the G modes are linked to the in plane correlation length inside the material

\footnotetext{
${ }^{2}$ http://www.ens-lyon.fr/LST/Raman
} 

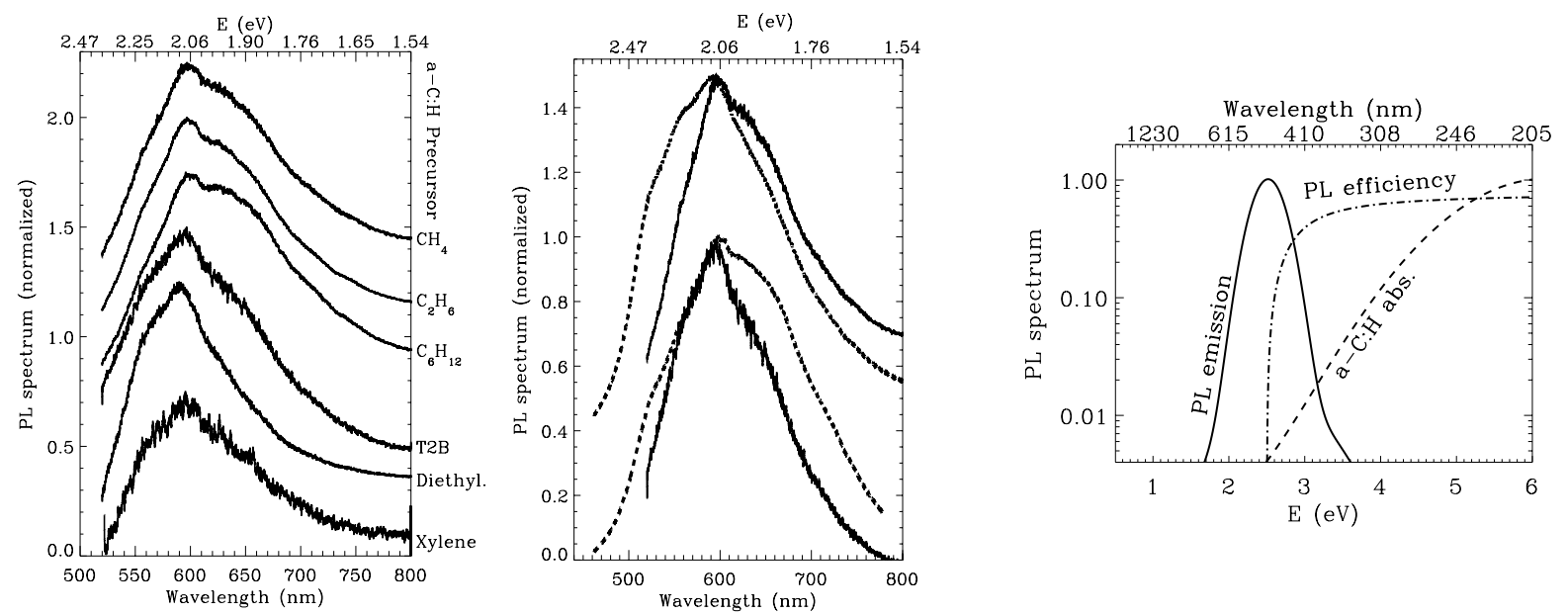

Fig. 10. Photoluminescence spectra. Left panel: photoluminescence spectra of photo-produced a-C:H polymer, at an excitation wavelength of $514.5 \mathrm{~nm}$ (green, $\sim 2.4 \mathrm{eV}$ ). Central panel: comparison of the photoluminescence spectra for photo-produced a-C:H prepared with methane (upper traces) and trans-2-butene (lower traces) precursors at two different excitation wavelength: $457.9 \mathrm{~nm}$ (blue, $\sim 2.7 \mathrm{eV}$, dashed lines) and $514.5 \mathrm{~nm}$ (green, $\sim 2.4 \mathrm{eV}$, full lines). Right panel: schematic view on the position and trend of photoluminescence efficiency and spectrum in a-C:H.

(Schwan et al. 1996). The G mode width of the a-C:H produced in our experiments exceed $50 \mathrm{~cm}^{-1}$, meaning that the correlation length lies below $1 \mathrm{~nm}$, a sign of a high "disorder".

\section{Photoluminescence}

As briefly mentioned in the previous section, Raman spectra are often difficult to interpret because of the photoluminescence (PL) background induced by the excitation laser. This drawback of Raman spectroscopy is however extremely interesting for a-C:H in view of its possible contribution to the Extended Red Emission spectrum (ERE), as already proposed for parent amorphous carbons (Duley 1985; Duley \& Williams 1990; Sakata et al. 1992; Webster 1993).

The photoluminescence spectra of the photo-produced a-C:H polymer are displayed in Fig. 10, left panel, obtained with an excitation wavelength of $514.5 \mathrm{~nm}$ (green, $\sim 2.4 \mathrm{eV}$ ). For a few of the polymers (with methane and trans-2-butene precursors), a previous spectrum had been recorded, with an excitation wavelength of $457.9 \mathrm{~nm}$ (blue, $\sim 2.7 \mathrm{eV}$ ), and the corresponding photoluminescence spectra are compared in the central panel of Fig. 10. Apart from a broadening of the line, the central wavelength of emission does not strongly shift or shifts slowly to higher energies. The photoluminescent a-C:H gives rise to a broad emission band around the optical gap of the material, excited by photons at or above this gap. A schematic diagram of the process is given in the right panel of Fig. 10. The center of the PL emission is correlated with this gap (Rusli et al. 1997; Robertson 1996a), but the position of the emission peak maximum does not vary by large amounts with the excitation energy (Koós et al. 1998), provided that this energy does not reach other gaps, like the $\mathrm{sp}^{3}$ bonds $(\sigma$ electronic states) above 5-6 eV. The photoluminescence efficiency of the PL emission has been shown to increase with the energy of the optical gap (Schütte et al. 1993; Robertson 1996b) which is correlated with the number of non-radiative recombination centers (Giorgis et al. 1998).

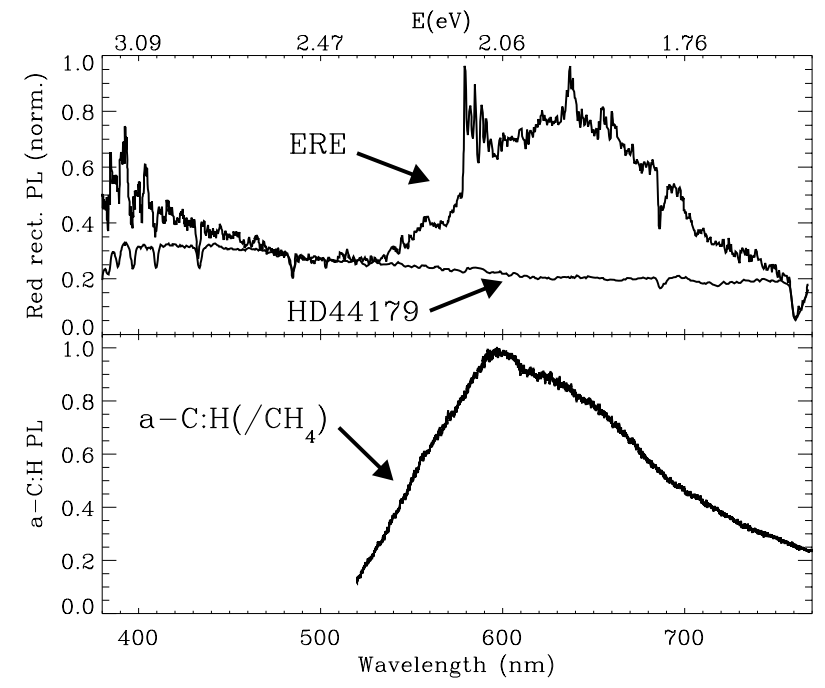

Fig. 11. Comparison between the Extended Red Emission (upper panel) and a-C:H photoluminescence spectrum (lower panel). The ERE spectrum was obtained by Schmidt et al. (1980) as well as a reference spectrum of the Red Rectangle central star (HD 44179) spectrum. The later has been scaled to the continuum level of the ERE, to highlight the excess emission. There is a fair agreement with the photoluminescence spectrum of photoproduced a-C:H. The small bump sumperimposed on the laboratory photoluminescent spectrum around $550 \mathrm{~nm}$ (weak) and $600 \mathrm{~nm}$ (more pronounced) are the Raman emission of the first and second harmonic of $\mathrm{C}=\mathrm{C}$ chemical groups in a$\mathrm{C}: \mathrm{H}$, and should be decoupled from the analysis.

A comparison between the photoluminescence spectrum of our photoproduced a-C:H and the Extended Red Emission spectrum from Schmidt et al. (1980) is given in Fig. 11 and shows a good agreement in the spectral profile. The small bump superimposed around $600 \mathrm{~nm}$ can be attributed to the second harmonic of the Raman $\mathrm{C}=\mathrm{C}$ active modes (Raman shift around $2800 \mathrm{~cm}^{-1}$ ), and the curve should be smoother. 

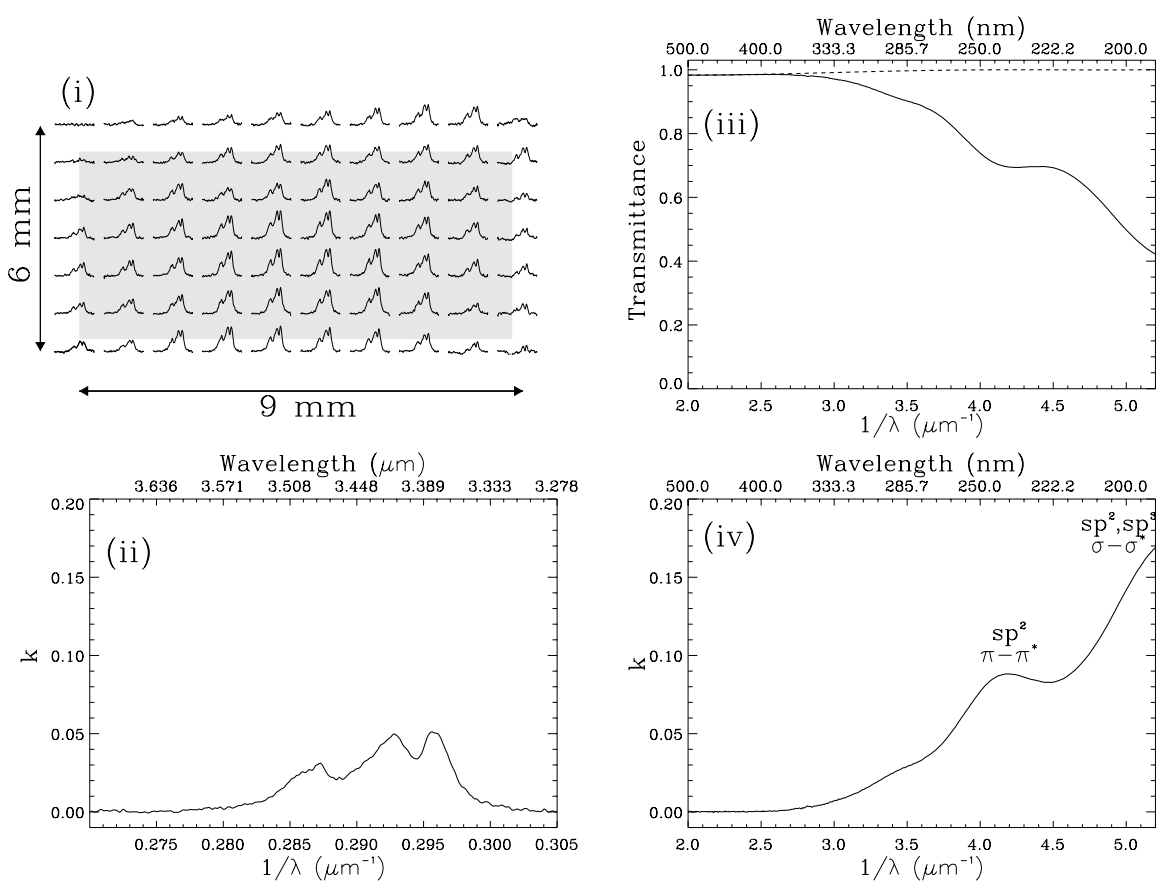

Fig. 12. From top to bottom, left to right: (i) infrared microscope optical depth ( $y$ axis of the individual spectra) map between 3050 and $2700 \mathrm{~cm}^{-1}$ ( $x$ axis of the individual spectra) of the $\mathrm{a}-\mathrm{C}: \mathrm{H}$ film measured in the visible-UV. Each spectrum is separated by $1 \mathrm{~mm}$ on the substrate. The grey zone represent the visible-UV spectrometer beam extend $(\sim 8 \mathrm{~mm} \times 5 \mathrm{~mm})$; (ii) mean imaginary part of the complex index of the material of the gray zone in the $\mathrm{CH}$ infrared stretching modes of the film; (iii) Visible-UV transmittance spectrum of the a-C:H film in the grey region; the dashed line represents a model of the transmittance of the $\mathrm{CaF}_{2}$ substrate plus film without absorption, used as the baseline for extraction; (iv) imaginary part of the complex index of the amorphous film, derived assuming the film is purely absorbant and determining the thickness from the infrared measurements, whose optical constants were previously derived.
The Raman spectrometer of the Laboratoire de Sciences de la Terre is not configured to allow quantitative photoluminescence measurements. However, to get a very crude order of magnitude for the photoluminescence efficiency of a-C:H, we also measured the PL of solid rodhamine 6G (Aldrich), a classical laser dye (with a PL efficiency of $100 \%$ when diluted in the liquid state), under the same illumination. Its integrated PL emission energy was only ten times higher than that of a-C:H, implying a high efficiency for a-C:H. In addition, we noted a fatiguing effect during the measurement, i.e. a decrease in photoluminescence efficiency with exposure time to the Raman laser, which is recovered when the laser exposure is stopped for a few minutes. This implies that the photoluminescence efficiency of our photoproduced material is even higher in an astrophysical context where photons are absorbed by a-C:H and relaxed one by one most of the time.

\section{UV-visible spectrum}

The a-C:H presented in this article must not only fulfills the requirement for infrared observations but also for UV-visible spectra. In particular, as discussed by e.g. Duley \& Seahra (1999), it is of high interest to understand the contribution of such material to the interstellar extinction curve. Any material that is produced in the laboratory must indeed be compatible with the full wavelength range to be considered as a good analog and viable candidate.

We were interested in evaluating the ratio of UV to infrared extinction of our photoproduced a-C:H. To do so, a thin layer of a-C:H produced from the hexane precursor was deposited onto a $\mathrm{CaF}_{2}$ transmitting window. Such a substrate is transparent both in the infrared $\mathrm{CH}$ stretching mode region and the UV-visible.
The transmittance of this film was measured with a CARY 5 spectrophotometer, from $190 \mathrm{~nm}$ to $1000 \mathrm{~nm}$ $(\sim 1.23-6.7 \mathrm{eV})$, at a resolution of $1 \mathrm{~nm}$, with a beam on the sample of about $8 \mathrm{~mm}$ height by $5 \mathrm{~mm}$. The same region on the substrate was mapped with the infrared microscope, in order to establish the corresponding absorption in the infrared $\mathrm{CH}$ stretching modes. The measured infrared microscope optical depth map between 3050 and $2700 \mathrm{~cm}^{-1}(3.27-3.7 \mu \mathrm{m})$ and corresponding visible-UV transmittance are displayed in Fig. 12, in the upper panels. The grey area on the infrared map corresponds to the extent of the visible-UV beam.

Using the optical constants obtained precedently, the film thickness can be established and used to derive the approximate visible-UV imaginary part $(k)$ of the refractive index of a-C:H, after modelling the transmittance of the $\mathrm{CaF}_{2}$ substrate. The expected $k$ index is presented in the lower panels of Fig. 12. The visible-UV spectrum presents two marked absorptions, around $4.2 \mu \mathrm{m}^{-1}$ and another one below the limit of the explored wavelenth range in the Far-UV. The first absorption is related to $\pi$ bonding of carbon atoms in the $\mathrm{sp}^{2}$ configuration, whereas the rise can be attributed to $\sigma$ bonding of carbon atoms in $\mathrm{sp}^{2} / \mathrm{sp}^{3}$ configurations. Unlike graphite, the $\mathrm{k}$ index of amorphous carbon material is expected to globally decrease as the energy decreases. This can be seen in the laboratory as the thin film presents a brown, yellowish colour when sufficiently thick to absorb enough visible light.

The maximum amplitude of $k$ in the $\pi \rightarrow \pi^{*}$ transition for graphite is about twenty times the a-C:H one. This is in agreement with the much lower amount of $\pi$ bonding (a-C:H network is amorphous) and the disorder in the electronic state (which makes the band wider). The position of the $k$ bump $\left(\sim 4.2 \mu \mathrm{m}^{-1}\right)$ lies at higher energy than for 
graphite $\left(\sim 3.65 \mu \mathrm{m}^{-1}\right)$, a blue shift already discussed by e.g. Logothetidis 2003.

The ratio of the a-C:H extinction in the UV bump to the $\mathrm{CH}$ infrared modes is to first order proportional to $3.4 \times$ $k(0.24 \mu \mathrm{m}) / 0.24 \times k(3.4 \mu \mathrm{m})$, i.e. about 30 . The same ratio estimated for the DISM in e.g. Duley \& Seahra (1999) is an order of magnitude higher.

These measurements allow us to give a first estimate of the contribution to the UV extinction of the photoproduced a-C:H. A more careful study in the Vis-UV must be undertaken to better constrain the dielectric function of this material. Several parameters will influence both the exact position of the bump, as well as its strength. We will soon study its dependence on the $\mathrm{sp}^{2}$ and $\mathrm{sp}^{3}$ fraction in the a-C:H network upon ion irradiation. Mennella et al. (2003) showed that the irradiation of HAC with energetic ions increases the strength of the so-called uv bump, probably because it starts to graphitize the material as the hydrogen atoms are ejected. This is indeed one of the subsequent modifications a-C:H experiences in space, an ingredient that has to be taken into account.

Modeling of an MRN distribution (Mathis et al. 1977) is essential to compare the extinction in the bump with interstellar observations. In particular, for graphite's extinction case, such a modeling strongly determines the position of the bump (see for example Fig. 6 in Draine \& Malhotra 1993). This is however specific to pure graphite, with its two principal axes and specific electronic properties. For an amorphous material such as $\mathrm{a}-\mathrm{C}: \mathrm{H}$, with its non-prefered axis (three dimensional network) and relatively low refractive index, the position of the bump as measured in the laboratory should lie closer to the observed interstellar one (e.g. Mennella et al. 1998). A recent study on laser production of amorphous carbon, followed by warming to $600 \mathrm{~K}$ (Duley \& Lazarev 2004) observed an excellent fit to the interstellar bump. The infrared spectrum of such material must also be investigated carefully in parallel and compared to the interstellar one, the ISM one being dominated by aliphatic features.

\section{$\mathrm{A}-\mathrm{C}: \mathrm{H}$ and the astrophysical context}

Photoproduced a-C:H films display an infrared spectrum that best reproduces the observational diffuse interstellar dust one, as described previously in a short communication (Dartois et al. 2004b). We have shown (Sect. 3) that a-C:H can be produced with the same chemical structure starting from many different organic precursors.

The resultant hydrogen and carbon network shares common properties that will depend principally on the original $\mathrm{H} / \mathrm{C}$ ratio and the amount of energy deposited in the system. Laboratory VUV photolysis is a soft $(\lesssim 10 \mathrm{eV})$ way to link together the basic units that build a-C:H. Therefore, plasma-produced a-C:H can be considered an extension of much higher energetic processing (sometimes not astrophysically relevant) of a common material.

The photo-produced a-C:H has been characterized by analysing both infrared and Raman laboratory spectra of the produced analogs. The so-called " $3.4 \mu \mathrm{m}$ absorption" profile has been decomposed into the various $\mathrm{sp}^{1}, \mathrm{sp}^{2}$ or $\mathrm{sp}^{3}$ bondings, and analysed. We stressed the influence of a Fermi resonance from methylene groups on this profile. In addition, neuronal network simulations have allowed us to confirm the organic structure of the underlying basic units of the photoproduced a-C:H polymer/disordered solid. Such simulations allow us to reject much more graphitic kerogen-like structures to explain both the a-C:H produced and the diffuse interstellar medium dust spectrum. We conclude that the carrier of the $3.4 \mu \mathrm{m}$ band and the mid-infrared diffuse interstellar medium features is no longer a poorly-defined organic structure.

Hydrogen-rich photo-produced or plasma-generated a-C:H always possess a $\mathrm{CH}_{2} / \mathrm{CH}_{3}$ ratio around 2 as monitored by IR spectra in the $\mathrm{CH}$ stretching mode window (e.g. the sequence of spectra in Ristein et al. 1998; this paper), with various total hydrogen atomic \% contents, up to the change to diamond-like $\mathrm{CH}$ character at low hydrogen content. This does not mean that there are no aromatic structures in the network, but that they are "invisible" in the $\mathrm{CH}$ stretching mode as these aromatics form clusters linked to the carbon network (see Fig. 5). The a-C: $\mathrm{H} \mathrm{CH}_{2} / \mathrm{CH}_{3}$ ratio is strikingly similar to the interstellar dust grain ratio. If a-C:H does not depart easily from this canonical value, it is because it must be driven by the needs for the growth of a stable network. For example, $\mathrm{CH}_{3}$ terminations are sterile, because hydrogen atoms passivates possible extensions of the network, and implies $\mathrm{CH}_{2} / \mathrm{CH}_{3}$ must be quite above 1 to be extended. On the other side of the ratio constraints, if the extension of a network made up of $\mathrm{C} \equiv \mathrm{C}, \mathrm{C}=\mathrm{C}$, $\mathrm{C}-\mathrm{C}$ and $\mathrm{C}-\mathrm{H}$ bonds is increased too much using only $\mathrm{CH}_{2}$ chains, the network is weakened at these chain locations, as the $\mathrm{CH}$ bonds are locally increased and thus possible $\mathrm{H}$ abstraction followed by the stitching of the chain with an unsaturated carbon atom enhanced. The space abundance ratio with $\mathrm{H} \gg \mathrm{C}$ would therefore drive the initial formation of $\mathrm{H}$-rich a-C:H whereas irradiation inhibits long chain solid polymers formation in the DISM. Subsequent evolution can of course drastically lower the polymer/disordered solid $\mathrm{H} / \mathrm{C}$ ratio, for instance, in media where atomic hydrogen is scarce.

The annealing experiments (Sect. 4), which mainly simulate the progressive hydrogen elimination from the a-C:H network, display a progressive evolution from a high aliphatic and low olefinic/aromatic network to aliphatic/aromatic and then almost purely aromatic network. An astrophysically important issue/consequence that we are currently investigating is the possibility that cosmic irradiation and/or heating of a-C:H could produce $\mathrm{H}_{2}$, even at low extinctions, without requiring the encounter and sticking of individual $\mathrm{H}$ atoms on a surface.

If we consider a starting a-C:H, with an $\mathrm{H} / \mathrm{C}$ ratio of $\approx 2$, that fully dehydrogenates to form a pure carbon particle, by e.g. thermal processing and/or high energy ( $\sim \mathrm{Mev})$ proton irradiation, then the upper limit on $\mathrm{H}_{2}$ production is one molecule per initial a-C:H carbon atom. However, this process could be recycled if protons can again saturate (hydrogenate) the carbon backbone. In order for this to occur, these protons must be of low energy $(\leq 100 \mathrm{keV})$ to be stopped within a network a few microns thick. Such amorphous grains would serve as catalysts for the formation of the first $\mathrm{H}_{2}$ molecules at the early stages of cloud condensations. 
We discussed the photoluminescence spectrum of a-C:H (Sect. 6) and its possible relation to the extended red emission. Many efforts have concentrated on producing a material able to fit such an emission in the laboratory (see references in Sect. 4 of the ERE review by Witt \& Vijh 2004). Based on the literature on a-C:H, we can take the inverse problem: if ERE is related to a-C:H, it gives some insight into its bandgap energy. ERE peaks generally appear between 600 and $750 \mathrm{~nm}$ (Darbon et al. 1999; Smith \& Witt 2002), i.e. between $1.65 \geq E_{\mathrm{PL}} \geq 2.1 \mathrm{eV}$, and therefore its optical gap should lie in the 1.7 to $2.5 \mathrm{eV}$ range (Rusli et al. 1997; Wagner \& Lautenschlager 1986; Yoshimi et al. 1992). It provides another constraint, based on the relation between the Tauc gap (Tauc et al. 1966) and the $\mathrm{sp}^{2}$ fraction in the network (Fig. 4 of Baptista \& Zawislak 2004), which should be between $20 \%$ (high optical gap) and 50\% (low optical gap). There might exist another fraction of DISM dust with a lower optical gap (higher $\mathrm{sp}^{2}$ content) but then it becomes unobservable by ERE, due to the rapid drop of the photoluminescence efficiency.

The interstellar dust seen in absorption must fulfil a given number of observational constraints: resist thermal evaporation, hard irradiation field (UV, protons), contain low oxygen content, display vibrational modes that are larger than simple molecules with a $\mathrm{CH}_{2} / \mathrm{CH}_{3}$ ratio around 2. Given the low ISM density, the collision timescale is too large to form a stable polymer or disordered solid in situ, and therefore favours production in the ejecta of stars followed by dissemination in the ISM.

Acknowledgements. We would like to thank B. Leriche, F. Langlet, N. Coron, J. Leblanc from IAS, S. Collin from CSNSM for helpful discussions and their help in optimizing the experiments. We also especially thank Anthony Jones for fruitful discussions. The authors are grateful to Gisèle Roger from the Laboratoire Charles Fabry de l'Institut d'Optique for measuring the visible-UV spectra.

\section{References}

Allen, D. A., \& Wickramasinghe, D. T. 1981, Nature, 294, 239

Baptista, D. L., \& Zawislak F. C. 2004, Diamond Relat. Mater., 13, 1791

Blanco, A., Bussoletti, E., Colangelli, L., Fonti, S., \& Stephens, J. R. 1991, ApJ, 382, L97

Bourée, J. E., Godet, C., Etemadi, R., \& Drévillon, B. 1996, Synthetic Metals 76, 191.

Butchart, I., McFadzean, A. D., Whittet, D. C. B., Geballe, T. R., \& Greenberg, J. M. 1986, A\&A, 154, L5

Chiar, J. E., Tielens, A. G. G. M., Whittet, D. C. B., et al. 2000, ApJ, 537, 749

Chiar, J. E., Adamson, A. J., Pendleton, Y. J., et al. 2002, ApJ, 570, 198

Darbon, S., Perrin, J., \& Sivan, J. 1999, A\&A, 348, 990

Dartois, E., Marco, O., Muñoz-Caro, G. M., et al. 2004a, A\&A, 423, 549

Dartois, E., Muñoz Caro, G. M., Deboffle, D., \& d'Hendecourt, L. 2004b, A\&A, 423, L33

Draine, B. T., \& Malhotra S. 1993, ApJ, 414, 632

Duley, W. W. 1994, ApJ, 430, L133

Duley, W. W. 1985, MNRAS, 215, 259.

Duley, W. W., \& Williams, D. A. 1983, MNRAS, 205, 67P

Duley, W. W., \& Williams, D. A. 1990, MNRAS, 247, 647
Duley, W. W., \& Seahra, S. S. 1999, ApJ, 522, L129

Duley, W. W., \& Lazarev, S. 2004, ApJ, 612, L33

Duley, W. W., Scott, A. D., Seahra, S., \& Dadswell, G. 1998, ApJ, 503, L183.

Ehrenfreund, P., Robert, F., D’Hendencourt, L., \& Behar, F. 1991, A\&A, 252, 712

Ferrari, A. C. 2002, Diamond Relat. Mater., 11, 1053.

Furton, D. G., Laiho, J. W., \& Witt, A. N. 1999, ApJ, 526, 752

Geballe, T. R., Chiar, J., Pendleton, Y. J., \& Tielens, A. G. G. M. 1997, Ap\&SS, 255, 457

Giorgis, F., Giulani, F., Pirri, C. F., Tagliaferro, A., \& Tresso, E. 1998, Appl. Phys. Lett., 72, 20, 2520

Grishko, V. I., \& Duley, W. W. 2002, ApJ, 568, 448

Hawkins, G. J. 1998, Spectral characterisation of infrared materials and filters, Thesis, The University of Reading, UK

Imanishi, M. 2000, MNRAS, 319, 331.

Jacob, W., \& Moller, W. 1993, Appl. Phys. Lett., 63, 13, 1771

Jones, T. J., Hyland, A. R., \& Allen, D. A. 1983, MNRAS, 205, 187

Jones, A. P., Duley, W. W., \& Williams, D. A. 1990, QJRAS, 31, 567

Jordanov, B., Tsankov, D., \& Korte, E. H. 2003, J. Molecular Structure, 651, 101

Koidl, P., Wagner, C., Dischler, B., Wagner, J., \& Ramsteiner, M. 1990, Mater. Sci. Forum 52, 41.

Koós, M., Pócsik, I., Erostyák, J., \& Buzádi, A. 1998, J. Non-Cryst. Solids, 227, 579

Lamberton, R. W., Morley, S. M., Maguire P. D., \& McLaughlin J. A. 1998, Thin Solid Films, 333, 114

Lazar, G., \& Lazar, I. 2003, J. Non-Cryst. Solids, 331, 70

Logothetidis, S. 2003, Diamond Relat. Mater., 12, 141

Manner, W. L., Bishop, A. R., Girolami, G. S., \& Nuzzo, R. G. 1998, J. Phys. Chem., 102, 8816

Mathis, J. S., Rumpl, W., \& Nordsieck, K. H. 1977, ApJ, 217, 425

Mathis, J. S., Mezger, P., \& Panagia, N. 1983, A\&A, 128, 212

McFadzean, A. D., Whittet, D. C. B., Bode, M. F., Adamson, A. J., \& Longmore, A. J. 1989, MNRAS, 241, 873

Mennella, V., Colangeli, L., Bussoletti, E., Palumbo, P., \& Rotundi, A. 1998, ApJ, 507, L177

Mennella, V., Brucato, J. R., Colangeli, L., \& Palumbo, P. 2002, ApJ, 569,531

Mennella, V., Baratta, G. A., Esposito, A., Ferini, G., \& Pendleton, Y. J. 2003, ApJ, 587, 727

Moore, M. H., \& Donn, B. 1982, ApJ, 257, L47

Motta, E. F., \& Pereyra, I. 2004 J. Non-Cryst. Solids, 338, 525

Nava, D., Rajmankina de Parada, T., Gonzalez, E., Boscan, N., De la cruz, C. 1996, Spectrochimica Acta A, 52, 1201

Pendleton, Y. J., \& Allamandola, L. J. 2002, ApJS, 138, 75

Pendleton, Y. J., Sandford, S. A., Allamandola, L. J., Tielens, A. G. G. M., \& Sellgren, K. 1994, ApJ, 437, 683

Ratajczak, H., \& Yaremko, A. M. 1998, Chem. Phys. Lett., 314, 122

Raynal, P. I., Quirico, E., Borg, J., et al. 2000, Planet. Space Sci., 48, 1329

Ristein, J., Stief, R. T., Ley, L., \& Beyer, W. 1998, J. Appl. Phys., 84, 3836

Robertson, J. 1995, Diamond Relat. Mater., 4, 297

Robertson, J. 1996a, Diamond Relat. Mater., 5, 457

Robertson, J. 1996b, J. Non-Cryst. Solids, 198, Part 2, 615

Robertson, J. 2002, Mater. Sci. Eng., 37, 129

Rusli, Robertson, J., \& Amaratunga, G. A. J. 1997 Diamond Relat. Mater., 6, 700

Sakata, A., Wada, S., Narisawa, T., et al. 1992, ApJ, 393, L83

Sandford, S. A., Allamandola, L. J., Tielens, A. G. G. M., et al. 1991, ApJ, 371, 607

Sandford, S. A., Pendleton, Y. J., \& Allamandola, L. J. 1995, ApJ, 440, 697 
Schütte, S., Will, S., Mell, H., \& Fuhs, W. 1993, Diamond Relat. Tauc, J., Grigorovici, R., \& Vancu, A. 1966, Phys. Status Solidi, 15, Mater., 2, 1360

Schmidt, G. D., Cohen, M., \& Margon, B. 1980, ApJ 239, L133

Schwan, J., Ulrich, S., Bathori, V., Ehrardt, H., \& Silva S. R. P. 1996, J. Appl. Phys., 80, 440

Scott, A. D., Duley, W. W., \& Jahani, H. R. 1997, ApJ, 490, L175

Selzer, P., Gasteiger, J., Thomas, H., \& Slazer, R. 2000, Chem. Eur. J., 6, 920

Smith, T., \& Witt, A. 2002, ApJ, 565, 304

Snow, T. P., \& Witt, A. N. 1996, ApJ, 468, L65

Snyder, R. G., \& Scherer, J. R. 1979, J. Chem. Phys., 71(8), 3221

Spoon, H. W. W., Keane, J. V., Tielens, A. G. G. M., et al. 2002, A\&A, 385,1022

Spoon, H. W. W., Armus, L., Cami, J., et al. 2004, ApJS, 154, 184

Swanepoel, R. 1983, J. Phys. E, 16, 1214 627

Tielens, A. G. G. M., Wooden, D. H., Allamandola, L. J., Bregman, J., \& Witteborn, F. C. 1996, ApJ, 461, 210

Vyskocil, J., Siroky, P., Vorlicek, V., \& Perina, V. 1996, Diamond Relat. Mater., 5, 466

Wagner, J., \& Lautenschlager, P. 1986, J. Appl. Phys., 59, 2044

Webster, A. 1993, MNRAS, 264, L1

Wexler, A. S. 1967, Appl. Spec. Rev., 1, 29

Witt, A. N., \& Vijh, U. P. 2004, in Astrophysics of Dust, ed. A. N. Witt, C. Clayton, \& B. T. Draine, ASP Conf. Ser., 309

Yoon, S. F., Rusli, Ahn, J., Zhang, Q., et al. 1999, Thin Solid Films, 340,62

Yoshimi, M., Shimizu, M., Hattori, K., \& Okamoto H. 1992, Optoelectronics, 7, 69 